\section{Confiabilidade do Sistema de Informações sobre Nascidos Vivos Hospitalares no Município do Rio de Janeiro, 1999-2001}

\author{
Reliability of birth certificate data \\ in Rio de Janeiro, Brazil, 1999-2001
}

Mariza Miranda Theme Filha 1 Silvana Granado Nogueira da Gama 2 Cynthia Braga da Cunha 3 Maria do Carmo Leal 2
1 Coordenação de Programas de Epidemiologia, Secretaria Municipal de Saúde do Rio de Janeiro, Rio de Janeiro, Brasil. 2 Departamento de Epidemiologia e Métodos Quantitativos em Saúde. Escola Nacional de Saúde Pública, Fundação Oswaldo Cruz, Rio de Janeiro, Brasil.

3 Instituto de Medicina

Social, Universidade do Estado do Rio de Janeiro, Rio de Janeiro, Brasil.

Correspondência Mariza Miranda Theme Filha Rua Afonso Cavalcanti 455, Rio de Janeiro, $R J$ 20211-110, Brasil. mtheme@pcrj.rj.gov.br

\section{Abstract}

The objective of this article is to assess coverage and reliability of data from the Information System on Live Births in the City of Rio de Janeiro, based on an analysis of live birth certificates issued by the various hospitals and compared to information from the "Study on Neonatal and Perinatal Morbidity and Mortality and Care in the City of Rio de Janeiro". A total of 9,608 interviews with post-partum women were paired case-by-case with the respective birth certificates. The statistical analysis consisted of calculating the Kappa index adjusted to the prevalence of categorical variables and the intra-class correlation coefficient for continuous variables, with a 95\% significance level. The study showed excellent coverage (96.5\%) for hospital births in the City of Rio de Janeiro during the period studied. The variables presenting the highest concordance levels (over 0.90) were the infant's sex, birth weight, mother's age, type of delivery, and type of pregnancy. The variables marital status, mother's schooling, and number of prenatal visits showed lower reliability indices (Kappa <0.70). The article discusses the importance of data quality in the Information System on Live Births (SINASC) and the system's use as a tool to help define maternal and child health policies.

Information Systems; Live Births; Birth Certificates

\section{Introdução}

Os eventos vitais são definidos como todos os acontecimentos que ocorrem na vida do indivíduo, alterando de alguma forma seu estado perante a sociedade, tendo grande importância jurídica. Em relação aos nascimentos, os eventos vitais comprovam prova de filiação, nacionalidade, idade e parentesco, entre outros. No Brasil, os registros de nascimentos, até o início da década de 90, estiveram baseados exclusivamente no Sistema de Registro Civil e compreendiam apenas os nascimentos que eram registrados em cartório, com níveis variáveis de sub-registro, de acordo com as regiões do País 1,2.

Entretanto, o reconhecimento da importância das informações sobre os nascimentos vivos para as estatísticas de saúde, epidemiologia e demografia levou o Ministério da Saúde do Brasil a implantar, em 1990, o Sistema de Informações sobre Nascidos Vivos (SINASC), cujo objetivo principal era conhecer o perfil epidemiológico dos nascimentos vivos, segundo variáveis como peso ao nascer, duração da gestação, tipo de parto, idade da mãe e número de partos 3 . Antes da implantação do SINASC, as informações epidemiológicas só podiam ser obtidas através de pesquisas amostrais 4 . Outros países já dispunham de sistema semelhante, sendo essas bases de dados importantes ferramentas no conhecimento dos resultados em saúde, assim como na análise de custo-efetivi- 
dade e na tomada de decisão para ações voltadas para grupos com necessidades específicas.

O SINASC é um sistema de informações de âmbito nacional, sob a responsabilidade das Secretarias Municipais e Estaduais de Saúde. Ele baseia-se nos dados contidos na Declaração de Nascido Vivo (DNV), documento oficial obrigatoriamente emitido pela unidade de saúde onde ocorreu o nascimento ${ }^{5}$. As informações contidas na DNV permitem caracterizar alguns aspectos dos nascimentos extremamente úteis para o planejamento de ações na área materno-infantil. Desde sua implantação, vários trabalhos têm sido produzidos, revelando o perfil dos nascimentos ocorridos em várias localidades do Brasil e a cobertura do sistema $2,3,6,7,8,9,10$. Além das análises descritivas, o SINASC permite o cálculo de indicadores de saúde diretamente, como o coeficiente de mortalidade infantil e seus componentes, o coeficiente de mortalidade materna, o coeficiente de mortalidade perinatal e as taxas de fecundidade e de natalidade, sem depender das estimativas populacionais (exceto nas regiões com muito baixa cobertura do sistema). Além disso, por dispor de informações comuns à Declaração de Óbito (DO), permite o cálculo de taxas específicas de mortalidade para menores de um ano, contribuindo, ainda, para a qualificação do Sistema de Informações sobre Mortalidade 5,11.

O SINASC foi implantado no Município do Rio de Janeiro em 1993, e a Secretaria Municipal de Saúde (SMS) avalia que sua cobertura é muito boa desde 19942 2. Nessa cidade, as informações sobre os nascimentos são sistematicamente analisadas e divulgadas pela SMS, subsidiando os diversos programas de saúde e informando às unidades que emitem DNV o perfil dos seus nascimentos. Isso permite que se tenha um conhecimento mais geral do perfil da cidade, chegando a níveis menores de análise, como áreas específicas da cidade e estabelecimentos de saúde. No período de 1999 a 2001, o Município do Rio de Janeiro registrou, através do SINASC, uma média de 100 mil nascimentos ao ano, dos quais $99,4 \%$ ocorreram em estabelecimentos de saúde. O predomínio quase absoluto dos nascimentos hospitalares tem seu paralelo na concentração desses nascimentos em algumas unidades de saúde. Em 2001, 84,3\% dos nascimentos ocorreram em 28 unidades, de um total de 180 que realizaram partos (http:// www.saúde.rio.rj.gov.br/).

A magnitude do SINASC e sua importância para a saúde pública em particular têm despertado a necessidade de avaliação das suas informações, seja do ponto de vista quantitativo (cobertura do sistema), seja do qualitativo (confia- bilidade das informações), de forma que os indicadores calculados reflitam realmente o perfil da população 5,12 .

Em 1999, foi iniciado o "Estudo da Morbimortalidade e da Atenção Peri e Neonatal no Município do Rio de Janeiro", que tinha como um de seus subprojetos a avaliação da confiabilidade das informações contidas nas DNVs emitidas pelas unidades hospitalares. Trata-se da primeira avaliação da qualidade do SINASC no município desde sua implantação em 1993.

O objetivo deste artigo é analisar a concordância dos dados do SINASC, confrontando-os com os dados obtidos pela pesquisa mediante duas fontes de informações, a entrevista com as puérperas e a pesquisa em prontuários.

\section{Metodologia}

\section{Desenho do estudo e coleta de dados}

O estudo da morbi-mortalidade e da atenção neo e perinatal no Município do Rio de Janeiro é um estudo seccional, realizado em uma amostra de puérperas que se hospitalizaram em maternidades por ocasião do parto, no período de julho de 1999 a março de 2001.

O plano amostral teve como unidades de sorteio os estabelecimentos de saúde, que foram agrupados em três estratos segundo características de risco neonatal (nascimento de bebês com baixo peso, considerado peso ao nascer inferior a $2.500 \mathrm{~g}$ ). Coincidentemente, os estratos se conformaram seguindo a divisão dos estabelecimentos de saúde conforme o tipo de prestador da assistência, uma vez que o risco neonatal segue essa distribuição. Formaramse, dessa forma, os seguintes estratos: estrato 1 , constituído por oito maternidades municipais e quatro federais; estrato 2, constituído por três maternidades militares, três estaduais, duas filantrópicas e duas privadas conveniadas com o SUS; estrato 3, composto por 25 maternidades privadas, totalizando uma amostra com 47 instituições. Dentro de cada estrato foi realizada uma amostragem em dois estágios: primeiro selecionou-se uma amostra dos estabelecimentos de saúde e depois, a amostra das puérperas em cada unidade escolhida. O tamanho da amostra em cada estrato foi estabelecido com o objetivo de comparar proporções em amostras de tamanhos iguais 13 no nível de significância de $5 \%$ e para detectar diferenças de pelo menos $3 \%$ com poder do teste de $90 \%$, baseando-se na proporção de baixo peso ao nascer, de acordo com os dados do SINASC do $\mathrm{Mu}$ nicípio do Rio de Janeiro em 1997. O total cal- 
culado para cada estrato foi de 3.500 puérperas (para maiores detalhes sobre a metodologia, ver Leal et al. 14).

A coleta de dados foi realizada mediante preenchimento de questionários específicos por acadêmicos bolsistas especialmente treinados para a pesquisa: um questionário para entrevista com a puérpera, outro para coleta de informações em prontuário e um terceiro com questões acerca da alta hospitalar da mãe e do bebê.

Para realização da análise de concordância entre as informações obtidas pela pesquisa e as constantes no SINASC, foi realizado o pareamento caso a caso de todas as entrevistas realizadas com as mães que deram à luz recém-nato vivo, com as respectivas DNVs constantes no SINASC, através da variável número da DNV. Quando este número não estava disponível nos dados da pesquisa, realizava-se a sua busca no banco do SINASC; para isso, utilizava-se a combinação de outras variáveis-chave, como nome da mãe, data de nascimento do bebê, unidade de saúde de nascimento e peso ao nascer, digitando-se, então, o número na base de dados da pesquisa, para realização do pareamento. Esse procedimento foi realizado em $20 \%$ dos casos.

\section{Análise dos dados}

Para fins de análise das variáveis categóricas, procedeu-se à compatibilização das categorias existentes no SINASC com as da pesquisa. A variável situação conjugal na entrevista apresentava apenas três categorias: solteira, casada e divorciada/separada/viúva. Como o SINASC apresentava cinco categorias, foi necessário somar as variáveis união consensual e casada, e separada judicialmente e viúva. A variável instrução da mãe foi coletada de forma aberta na entrevista, em anos completos de estudos concluídos. No SINASC, esta variável apresenta a mesma lógica, porém vem categorizada em cinco grupos (nenhuma, 1 a 3, 4 a 7, 8 a 11 e 12 e mais), o que nos obrigou a agrupar a variável da pesquisa também em cinco categorias. A variável idade gestacional se apresenta no SINASC em seis categorias, de acordo com as semanas de gestação (<22, 22-27, 28-31, 32-36, 37-41 e 42 e mais). Na entrevista com a mãe, a variável era registrada, perguntado-se com quantas semanas de gravidez a puérpera estava no momento do parto. Na pesquisa em prontuário, deveria ser registrada a idade gestacional em semanas, informando se a fonte era a data da última menstruação (DUM) ou ultra-sonografia. Nesta situação também procedemos à categorização da variável nas mesmas categorias do SINASC. As variáveis tipo de parto e tipo de gravidez apresentavam categorizações diferentes nos vários instrumentos, o que nos levou a realizar os agrupamentos de forma a compatibilizá-los. A variável tipo de parto foi agrupada em duas categorias (vaginal e cesáreo), e o tipo de gravidez, em três categorias (única, dupla, tripla ou mais). Em relação ao número de filhos tidos, nascidos vivos ou mortos, agrupamos em cinco categorias (nenhum, um, dois, três e quatro e mais). Quanto ao índice de Apgar, mantivemos as 11 categorias possíveis (variando de zero a dez). As variáveis idade da mãe e peso ao nascer apresentavam-se como variáveis contínuas em todos os formulários, e assim foram mantidas.

Utilizou-se o coeficiente Kappa ajustado à prevalência para avaliação da concordância de variáveis categóricas (escolaridade da mãe, estado civil, idade gestacional, número de consultas de pré-natal, tipo de parto, tipo de gestação, idade gestacional, sexo do recém-nascido, índice de Apgar no 1o e 5o minutos, número de filhos nascidos vivos e mortos e presença de anomalias congênitas) com nível de significância de 95\%. Para interpretação da magnitude dos valores de Kappa, utilizou-se a seguinte classificação: concordância quase perfeita para o Kappa entre 0,81 e 1,00; concordância excelente para o coeficiente entre 0,61 e 0,80 ; concordância moderada para índices entre 0,41 e 0,60 ; concordância sofrível para índices entre 0,21 e 0,40 e concordância fraca para índices abaixo de 0,20 15 .

Para medida da concordância das variáveis contínuas (idade da mãe e peso ao nascer), foi utilizado o Coeficiente de Correlação Intraclasse (Intraclass Correletion Coefficient - ICC), assumindo-se o caráter não aleatório dos entrevistadores (Two way mixed effect model) 16.

A análise foi realizada considerando-se o conjunto das entrevistas dos três estratos, informando-se a origem da informação na pesquisa (entrevista ou prontuário).

\section{Resultados}

O banco de dados para análise da concordância contava com 9.953 questionários respondidos pelas puérperas, após a exclusão dos casos em que o desfecho foi óbito fetal, uma vez que só nos interessavam os nascimentos vivos. Foi possível o pareamento de 9.608 questionários (proporção de pareamento de 96,5\%). As causas de não-pareamento foram a ausência do número da DNV em 43 questionários, mesmo após a busca no SINASC através das variáveischave, e a inexistência na base do SINASC de 
302 casos. Assim, verificamos um sub-registro no SINASC de 3,5\% dos nascimentos hospitalares. Vale ressaltar que a análise englobou todos os nascimentos ocorridos na cidade, de residentes e não residentes no Município do Rio de Janeiro.

Tanto a pesquisa perinatal quanto o SINASC apresentaram baixo percentual de informações ignoradas (menor que 10\%). Apenas as variáveis número de consultas de pré-natal e idade gestacional, obtidas através dos prontuários médicos, apresentaram elevada proporção de ausência de informações (respectivamente $47 \%$ e 30,2\%). Já no SINASC, as variáveis número de filhos nascidos vivos, número de filhos nascidos mortos e presença de anomalias congênitas mostraram altas freqüências de não informação, respectivamente $34,6 \%, 57,5 \%$ e $11,7 \%$ (Tabela1).

Quanto às variáveis relativas a gestação e parto, verificou-se elevado percentual de pareamento entre as variáveis dos dois sistemas (acima de 95\%), com exceção para idade gestacional quando a fonte de dados era o prontuário (pareamento de $70 \%$ ).

Na Tabela 2, a variável tipo de parto apresentou concordância quase perfeita, com Kappa ajustado de 0,97 para as fontes de informação entrevista e SINASC. Esse índice manteve-

Tabela 1

Proporção de ausência de informações das variáveis analisadas, segundo fonte de informação.*

\begin{tabular}{lcc}
\hline Variáveis & Pesquisa & SINASC \\
\hline Estado civil da mãe (E) & 0,1 & 3,9 \\
Idade da mãe (E) & 0,0 & 0,4 \\
Escolaridade da mãe (E) & 0,4 & 5,2 \\
Número de filhos nascidos vivos (E) & 0,1 & 34,6 \\
Número de filhos nascidos mortos (E) & 0,1 & 57,5 \\
Número de consultas pré-natal (E) & 4,6 & 2,7 \\
Número de consultas pré-natal (P) & 47,0 & - \\
Tipo de parto (E) & 0,2 & 0,5 \\
Tipo de parto (P) & 1,6 & - \\
Tipo de gestação (P) & 3,7 & 0,2 \\
Idade gestacional (E) & 0,0 & 0,8 \\
Idade gestacional (P) & 30,2 & - \\
Sexo do recém-nascido (P) & 2,6 & 0,0 \\
Peso ao nascer (P) & 2,3 & 0,5 \\
Apgar 1o minuto (P) & 9,1 & 0,9 \\
Apgar 5o minuto (P) & 9,0 & 0,7 \\
Anomalia congênita (P) & 6,1 & 11,7 \\
\hline
\end{tabular}

* $\mathrm{E}=$ Entrevista com a puérpera; $\mathrm{P}=$ Prontuário médico;

SINASC = Sistema de Informação sobre Nascidos Vivos. se elevado também para as comparações prontuário/SINASC e prontuário/entrevista, revelando que todas as fontes de informação apresentam alta confiabilidade. A variável tipo de gravidez também apresentou concordância quase perfeita após ajustamento do índice Kappa $(K=0,99)$. Para a variável idade gestacional, a confiabilidade das informações variou de quase perfeita a excelente, de acordo com a fonte de dados utilizada, com o coeficiente Kappa ajustado igual a 0,83 (prontuário/SINASC) e 0,73 (entrevista/SINASC).

Para as variáveis relativas às características da mãe, foi possível o pareamento segundo as diversas fontes de informação em mais de $90 \%$ para todas as variáveis, exceto para o número de consultas de pré-natal, quando esta dependia da informação do prontuário (pareamento apenas para metade dos casos), e para o número de filhos nascidos vivos e mortos. Entretanto, apesar da elevada taxa de pareamento para situação conjugal, a concordância foi apenas moderada (Kappa = 0,53). As variáveis número de filhos nascidos vivos e número de filhos nascidos mortos, apesar da menor proporção de pareamento, apresentaram concordância elevada através do Kappa ajustado (respectivamente 0,85 e 0,82). Já a variável instrução da mãe apresentou concordância excelente (Kappa = 0,66) (Tabela 3).

As variáveis relativas ao recém-nascido, além de alta taxa de pareamento, apresentaram excelente confiabilidade. O índice Kappa foi de 0,94 para a variável sexo e 0,97 para a variável anomalia congênita. Ambas as informações foram pareadas com o SINASC, tendo como fonte de pesquisa o prontuário médico. $\mathrm{O}$ mesmo podemos dizer em relação aos índices de Apgar nos 1o e 5o minutos, que também apresentaram concordância quase perfeita, com valores de Kappa ajustado à prevalência de 0,89 e 0,90 , respectivamente (Tabela 3 ).

Observa-se, na Tabela 4, a análise das variáveis contínuas através do ICC, mostrando excelente concordância para as variáveis idade da mãe $($ ICC $=0,980)$ e peso ao nascer (ICC = 0,966).

\section{Discussão}

O SINASC constitui-se atualmente em um instrumento extremamente valioso para análise da situação do nascimento e parto, bem como das características das mães e dos bebês, permitindo acompanhar o perfil deste segmento da população e traçar políticas de saúde adequadas, voltadas para grupos específicos, de 
Tabela 2

Concordância entre as variáveis relativas à gestação e ao parto, segundo a Pesquisa e o SINASC.

Município do Rio de Janeiro, Brasil, 1999-2001.

\begin{tabular}{lccccc}
\hline Variáveis & $\mathbf{n}$ & $\begin{array}{c}\text { Índice Kappa } \\
\text { bruto }\end{array}$ & p-valor & $\begin{array}{c}\text { IC95\% } \\
\text { Kappa ajustado } \\
\text { a prevalência }\end{array}$ \\
\hline Tipo de parto (entrevista/SINASC) & 9.542 & 0,97 & 0,000 & $0,96-0,97$ & 0,97 \\
Tipo de parto (prontuário/SINASC) & 9.414 & 0,95 & 0,000 & $0,95-0,96$ & 0,95 \\
Tipo de parto (entrevista/prontuário) & 9.436 & 0,97 & 0,000 & $0,96-0,97$ & 0,97 \\
Tipo de gravidez (prontuário/SINASC) & 9.242 & 0,74 & 0,000 & $0,68-0,80$ & 0,99 \\
Idade gestacional (entrevista/SINASC) & 9.532 & 0,27 & 0,000 & $0,25-0,30$ & 0,73 \\
Idade gestacional (prontuário/SINASC) & 6.655 & 0,41 & 0,000 & $0,38-0,44$ & 0,83 \\
Idade gestacional (prontuário/entrevista) & 6.704 & 0,39 & 0,000 & $0,37-0,42$ & 0,73 \\
\hline
\end{tabular}

SINASC = Sistema de Informação sobre Nascidos Vivos.

Tabela 3

Concordância entre as variáveis relativas às características da mãe e do recém-nato.

Município do Rio de Janeiro, Brasil, 1999-2001.

\begin{tabular}{|c|c|c|c|c|c|}
\hline Variáveis & n & $\begin{array}{l}\text { Índice Kappa } \\
\text { bruto }\end{array}$ & p-valor & IC95\% & $\begin{array}{l}\text { Kappa ajustado } \\
\text { à prevalência }\end{array}$ \\
\hline Situação conjugal (entrevista/SINASC) & 9.230 & 0,33 & 0,000 & $0,31-0,35$ & 0,53 \\
\hline Instrução da mãe (entrevista/SINASC) & 9.076 & 0,61 & 0,000 & $0,60-0,63$ & 0,66 \\
\hline $\begin{array}{l}\text { Número de consultas pré-natal } \\
\text { (entrevista/SINASC) }\end{array}$ & 8.922 & 0,48 & 0,000 & $0,47-0,50$ & 0,62 \\
\hline $\begin{array}{l}\text { Número de consultas pré-natal } \\
\text { (prontuário/SINASC) }\end{array}$ & 4.992 & 0,50 & 0,000 & $0,48-0,52$ & 0,57 \\
\hline Número de filhos nascidos vivos & 6.280 & 0,83 & 0,000 & $0,82-0,84$ & 0,85 \\
\hline Número de filhos nascidos mortos & 4.082 & 0,18 & 0,000 & $0,14-0,21$ & 0,82 \\
\hline $\begin{array}{l}\text { Número de consultas pré-natal } \\
\text { (prontuário/entrevista) }\end{array}$ & 4.804 & 053 & 0,000 & $0,51-0,55$ & 0,60 \\
\hline Sexo do recém-nascido (prontuário/SINASC) & 9.358 & 0,92 & 0,000 & $0,91-0,93$ & 0,94 \\
\hline Anomalia congênita (prontuário/SINASC) & 8.014 & 0,42 & 0,000 & $0,34-0,49$ & 0,97 \\
\hline Apgar 1o minuto (prontuário/SINASC) & 8.686 & 0,85 & 0,000 & $0,84-0,86$ & 0,89 \\
\hline Apgar 5 o minuto (prontuário/SINASC) & 8.701 & 0,85 & 0,000 & $0,84-0,86$ & 0,90 \\
\hline
\end{tabular}

SINASC = Sistema de Informação sobre Nascidos Vivos.

Tabela 4

Concordância entre as variáveis da Pesquisa e do SINASC através do ICC.

Município do Rio de Janeiro, Brasil, 1999-2001.

\begin{tabular}{lcccc}
\hline Variáveis & $\mathbf{n}$ & ICC & IC95\% & p-valor \\
\hline Idade da mãe (entrevista/SINASC) & 9.565 & 0,981 & $0,980-0,982$ & 0,000 \\
Peso ao nascer (prontuário/SINASC) & 9.344 & 0,966 & $0,965-0,967$ & 0,000 \\
\hline
\end{tabular}

SINASC = Sistema de Informação sobre Nascidos Vivos; ICC = Coeficiente de Correlação Intraclasse. 
acordo com perfil de risco. No entanto, como todo sistema de informações, é necessário conhecer em que medida as informações fornecidas são realmente confiáveis.

Em primeiro lugar, verificamos que a cobertura do SINASC no Município do Rio de Janeiro é bastante elevada (96,5\%). Estudo realizado em São Luiz, Maranhão, revelou cobertura estimada de $75,8 \% 12$. Em nosso município, de maneira geral, o SINASC apresenta baixas taxas de não-informação, com exceção para as variáveis número de filhos nascidos vivos e número de filhos nascidos mortos. Embora seja uma informação de grande valor quanto ao aspecto demográfico, para o acompanhamento contínuo e facilmente disponível da taxa de fecundidade, parece haver pouca conscientização, ou treinamento, sobre o seu correto preenchimento. Considerando que essa informação faz parte da rotina de entrevista com a mãe no momento da admissão na maternidade, que deve ser anotada, obrigatoriamente, no prontuário e que é informada pela mãe em quase $100 \%$ casos, fica a impressão de que se trata realmente de pouca valorização da informação como fonte de conhecimento real. Contudo, quando preenchida, ela teve excelente confiabilidade.

$\mathrm{O}$ mesmo podemos dizer a respeito da qualidade do registro no prontuário médico quanto à informação sobre número de consultas de pré-natal. Sabe-se que a realização de pré-natal é fator de proteção para desfechos neonatais negativos, como baixo peso, prematuridade, óbito pré-natal 17, e a ausência dessa informação nos prontuários impede o conhecimento sobre grupos populacionais mais vulneráveis. Ainda que pequena, surpreende a diferença na presença da informação dessa variável entre o prontuário, fonte de dados para o preenchimento da DNV, e as próprias DNVs dos nascimentos hospitalares analisados. Parece que, ao preencher a informação na DNV, utiliza-se a informação verbal não registrada, já que na análise das entrevistas com a mãe o índice de resposta foi alto. A variável número de consultas de pré-natal apresentou problemas tanto do ponto de vista quantitativo, quanto qualitativo. A confiabilidade da informação foi considerada apenas boa (Kappa $=0,62$ ) no pareamento do SINASC com as informações fornecidas pela mãe.

Dobie et al. 18 , em pesquisa realizada com 1.937 gestantes de baixo risco no Estado de Washington, Estados Unidos, encontrou os mesmos problemas em relação à precisão das informações sobre pré-natal. A análise baseada no pareamento dos dados da pesquisa com os certificados de nascimento revelou que estes subestimam o número de consultas de pré-natal e sugere que as informações devem ser utilizadas com cautela na alocação de recursos destinados às políticas de saúde. Outros estudos também mostram que a informação sobre número de consultas de pré-natal realizadas é pouco confiável 12,19,20.

A variável idade gestacional apresentou também problemas de registro em prontuário, embora tanto a entrevista com a mãe, como os dados do SINASC mostrassem percentuais elevados de informação. Porém, contrariamente ao pré-natal, a confiabilidade dos dados foi melhor. Isto é, quando registrada no prontuário, a informação é de excelente qualidade (Kappa ajustado $=0,73$ e 0,83). Em estudo sobre a validação dos certificados de nascimentos em New Jersey, Estados Unidos, no período de 19891992, Reichman \& Hade 20 encontraram alguma perda de concordância na avaliação da idade gestacional. Mello-Jorge 5 encontrou um nível de concordância de $80,7 \%$ para idade gestacional, e, em seu estudo, também os prontuários médicos apresentavam elevado percentual de ausência de informação (10,8\%). Uma hipótese é que a possibilidade de implicações médicas imediatas no momento do nascimento, exigindo tomada de decisões rápidas e/ou realização de procedimentos específicos, determinem maior confiabilidade da idade gestacional. Este fato também já havia sido destacado por Moraes \& Reichenheim 21 ao analisarem a concordância entre a identificação da prematuridade, tendo como base a DUM registrada no prontuário, e a informação baseada nas recordações da mãe no momento da entrevista. Já Silva et al. 12 observaram em seu estudo haver uma tendência, ao se preencher o campo idade gestacional na DNV, de se atribuir a categoria 37 a 41 semanas para crianças aparentemente a termo. Dessa forma, estariam sendo considerados prematuros somente aqueles recém-nascidos indiscutivelmente prematuros, o que levaria a uma subestimação dos prematuros limítrofes (idade gestacional entre 35 e 36 semanas).

As variáveis que apresentaram maiores índices de concordância (Kappa ajustado à prevalência e ICC maior que 0,95 ) foram tipo de parto, tipo de gravidez, idade da mãe, peso ao nascer e presença de anomalias congênitas.

Assim como a prematuridade, a qualidade do registro do peso ao nascer e da presença de anomalias congênitas tem uma lógica calcada na possibilidade do uso de tecnologia médica, podendo implicar a adoção de medidas imediatas (necessidade de suporte intensivo, procedimentos cirúrgicos, etc), o que faz com que 
esse registro seja valorizado pelo médico. Além de apresentar baixo sub-registro, sua confiabilidade é excelente. O ICC para peso ao nascer foi de 0,966 (IC: 0,965-0,967) e o coeficiente Kappa ajustado para a presença de anomalias congênitas foi igual a 0,97 . Diversos outros autores apontam para a excelente qualidade da informação sobre peso ao nascer, e, em alguns casos, a comparação entre a média de pesos obtida dos certificados de nascimentos e a dos prontuários médicos apresenta informações virtualmente idênticas 19,20,22.

Vários estudos têm mostrado que a confiabilidade das informações sobre dados demográficos da mãe (idade, escolaridade e situação conjugal) e tipo de parto e de gravidez é bastante elevada 19,20,22,23. Na nossa pesquisa encontramos concordância excelente para idade da mãe, tipo de gravidez e de parto. Em relação ao tipo de parto, a confiabilidade foi alta e independente da fonte de dados utilizada. Entre as variáveis maternas analisadas, a situação conjugal foi a que apresentou a menor concordância (Kappa $=0,53$ ). Um dos principais problemas detectados foi em relação ao tipo de informação aferida em cada documento. Na DNV a informação registrada é o estado civil, que tem conotação legal. Na pesquisa, a informação registrada era situação conjugal, que aborda mais especificamente a questão do suporte familiar (se tem ou não companheiro e se vive ou não junto). Essas duas maneiras distintas de aferição indubitavelmente tiveram grande influência sobre a concordância dos dados. Além disso, as diferentes formas de categorização dessa variável na pesquisa e no SINASC obrigaram a agregação de alguns componentes, que podem também ter contribuído para distorção dos dados.

A informação sobre a escolaridade materna apresentou boa concordância (Kappa $=0,66$ ), resultado que consideramos bastante satisfatório. Em primeiro lugar, por tratar-se de uma variável de difícil preenchimento, pois obriga quem está preenchendo a DNV a calcular o número de anos de estudos e escolher a casela correta. Em segundo lugar, porque somente a partir de julho de 1999 essa variável passou a ser classificada dessa forma, em substituição à antiga classificação, que contemplava os períodos escolares (1o grau, 2o grau e 3o grau). Alguns estudos têm mostrado que o uso do preenchimento de variáveis fechadas (uso de caselas) dá uma falsa sensação de segurança, quando comparado ao uso de respostas abertas 20. Em relação ao SINASC, acreditamos que essa é uma das possibilidades importantes de erro e que merece ser discutida. Além disso, as novas categorias não têm paralelo com a antiga classificação, impedindo a comparação com a série histórica desde a implantação do sistema, em 1990. Em sua pesquisa, Mello-Jorge et al. 5 também consideraram a escolaridade materna como variável de difícil obtenção, não constando geralmente seu registro nos prontuários (ausência de informação em 64,8\% destes). Esse fato teve influência significativa no índice de concordância entre o SINASC e a pesquisa em prontuário, que foi da ordem de $29,0 \%$.

A informação sobre o sexo do recém-nascido e índices de Apgar nos 1o e 5o minutos mostrou excelentes níveis de concordância, fato este destacado por outros autores 19,23. Todavia, estudos realizados no Brasil mostraram, em relação ao índice de Apgar, níveis relativamente mais baixos de concordância 5 .

\section{Considerações finais}

Esta pesquisa revestiu-se de grande importância por permitir avaliar vários aspectos da atenção peri e neonatal. Em relação ao subprojeto de Avaliação do Sistema de Informações sobre Nascidos Vivos, algumas considerações devem ser feitas.

Em primeiro lugar, destacamos a alta cobertura do SINASC e qualidade da maioria das informações. Com exceção das variáveis situação conjugal e número de consultas de pré-natal, as informações registradas apresentaram excelente concordância com os dados coletados na pesquisa.

Coerente com a literatura $4,5,12$, nossa pesquisa corrobora os dados nacionais. Assim, as variáveis número de consultas de pré-natal realizadas, número de partos e situação conjugal, que apresentaram os piores desempenhos do ponto de vista da confiabilidade dos dados, necessitam ser revistas pelos gestores do sistema, na busca de soluções que visem a melhorar a qualidade das informações. Essa melhoria passa necessariamente pelo treinamento dos profissionais que preenchem a DNV, mostrando-lhes a importância dessas informações para a saúde pública da nossa cidade ${ }^{4}$. Eles devem estar cientes de que são os indicadores calculados com base no SINASC que irão definir quais investimentos deverão ser aplicados na área materno-infantil. São, na sua maioria, indicadores que utilizam variáveis associadas a maior risco para desfechos neonatais negativos. Essa visibilidade só será alcançada com a ampla divulgação e democratização das informações geradas a partir de bancos de dados que são públicos, e, portanto, de acesso irrestrito. 
Este estudo mostrou também o enorme potencial da utilização de bases de dados que podem ser trabalhadas conjuntamente através do cruzamento de informações procedentes de várias origens (inquéritos de saúde, informações hospitalares, sistemas de informações sobre mortalidade), tornando-se excelente fonte de dados para a pesquisa epidemiológica e estudos sobre atenção perinatal. Entretanto, reiteramos que se faz necessário o investimento intensivo na qualificação de suas informações.

\section{Resumo}

O objetivo deste artigo é avaliar a cobertura e a confiabilidade das variáveis constantes no Sistema de Informações sobre Nascidos Vivos no Município do Rio de Janeiro, valendo-se da análise das Declarações de Nascidos Vivos emitidas pelas unidades hospitalares, confrontadas com as informações obtidas do "Estudo da Morbi-mortalidade e da Atenção Peri e Neonatal no Município do Rio de Janeiro". Foram pareadas 9.608 entrevistas realizadas com as puérperas e as respectivas DNVs (pareamento caso a caso). A análise estatística consistiu no cálculo do índice Kappa ajustado à prevalência para variáveis categóricas e no Coeficiente de Correlação Intraclasse para as variáveis contínuas, com nível de significância de 95\%. Verificou-se excelente cobertura dos nascimentos hospitalares ocorridos no Município do Rio de Janeiro, no período em estudo (96,5\%). As variáveis que apresentaram maiores concordâncias (acima de 0,90) foram sexo do recém-nascido, peso ao nascer, idade da mãe, tipo de parto e tipo de gestação. As variáveis situação conjugal, instrução da mãe e número de consultas de prénatal apresentaram índices de confiabilidade mais baixos (Kappa <0,70). Discute-se a importância da qualidade das informações do SINASC e o seu uso como instrumento de definição de políticas na área materno-infantil.

Sistemas de Informações; Nascidos Vivos; Declaração de Nascimento

\section{Colaboradores}

M. M. Theme Filha foi responsável por unir o banco de dados, pela análise e interpretação dos dados e pela redação do artigo. S. G. N. Gama colaborou na análise dos dados, redação do artigo e busca de referências bibliográficas. C. B. Cunha cooperou em todas as fases de limpeza e análise do banco de dados, bem como na elaboração e redação da metodologia do artigo. M. C. Leal participou na construção dos objetivos e em todas as fases de análise dos resultados obtidos. Contribuiu na redação e revisão final do artigo. 


\section{Referências}

1. Mello-Jorge MHP. Registro dos eventos vitais: sua importância em saúde pública. São Paulo: Centro Brasileiro de Classificação de Doenças, Faculdade de Saúde Pública, Universidade de São Paulo; 1990.

2. Silva RI, Theme Filha MM, Noronha CP. Sistema de Informações sobre Nascidos Vivos na cidade do Rio de Janeiro 1993/1996. Inf Epidemiol SUS 1997; 2:34-48.

3. Mello-Jorge MHP, Gotlieb SLD, Oliveira H. O Sistema de Informações sobre Nascidos Vivos: primeira avaliação dos dados brasileiros. Inf Epidemiol SUS 1996; 2:15-48.

4. Carvalho DM. Grandes sistemas nacionais de informação em saúde: revisão e discussão atual. Inf Epidemiol SUS 1997; 4:7-46.

5. Mello-Jorge MHP, Gotileb SLD, Soboll MLMS, Almeida MF, Latorre MRDO. Avaliação do Sistema de Informações sobre Nascidos Vivos e o uso de seus dados em epidemiologia e estatísticas de saúde. Rev Saúde Pública 1993; 27 Suppl:1-44.

6. Rodrigues CS, Magalhães Junior HM, Evangelista PA, Ladeira RM, Laudares S. Perfil dos nascidos vivos no Município de Belo Horizonte, 1992-1994. Cad Saúde Pública 1997; 13:53-7.

7. Maia MAC. Caracterização dos nascidos vivos hospitalares no primeiro ano de implantação do Subsistema de Informações sobre Nascidos Vivos, em município de Minas Gerais, Brasil, 1996. Rev Saúde Pública 1997 31:581-5.

8. D’Orsi E, Carvalho MS. Perfil de nascimentos no Município do Rio de Janeiro: uma análise espacial. Cad Saúde Pública 1998; 14:367-79.

9. Mello-Jorge MHP, Gotlieb SLD, Andrade SM. Análise dos registros de nascimentos vivos em localidade urbana no Sul do Brasil. Rev Saúde Pública 1997; 31:78-89.

10. Santa Helena ET, Wisbeck J. Implantação do SINASC e perfil dos nascidos vivos de Blumenau, 1994-1997. Inf Epidemiol SUS 1998; 3:35-42.

11. Noronha CP, Silva RI, Theme Filha MM. Concordância de dados das Declarações de Óbitos e de Nascidos Vivos para a mortalidade neonatal no Município do Rio de Janeiro. Inf Epidemiol SUS 1997; 4:57-65.
12. Silva AAM, RibeiroVS, Borba Junior AF, Coimbra LC, Silva RA. Avaliação da qualidade dos dados do Sistema de Informações sobre Nascidos Vivos em 1997-1998. Rev Saúde Pública 2001; 35:508-14.

13. Fleiss JL. Statistical methods for rates and proportions. New York: John Wiley \& Sons; 1981.

14. Leal MC, Gama SGN, Campos MR, Cavalini LT, Garbayo LS, Brasil CLP, et al. Fatores associados à morbi-mortalidade perinatal em uma amostra de maternidades públicas e privadas no Município do Rio de Janeiro, 1999-2001. Cad Saúde Pública 2004; 20 Suppl 1:S20-33.

15. Dunn G. Design and analysis of reliability studies. New York: John Wiley \& Sons; 1989.

16. Shrout PE, Fleiss JL. Intraclass correlation: Uses in assessing rater reliability. Psychol Bull 1979; 86:420-8.

17. Gama SGN, Szwarcwald CL, Leal MC. Experiência de gravidez na adolescência, fatores associados e resultados perinatais entre puérperas de baixa renda. Cad Saúde Pública 2002; 18:153-61.

18. Dobie AS, Baldwin LM, Rosenblatt RA, Foryce MA, Andrilla CH, Hart LG. How well do birth certificates describe the pregnancies they report? The Washington State esperience with low-risk pregnancies. Matern Child Health J 1998; 2:145-54.

19. Buescher PA, Taylor KP, Davis MH, Bowling JM. The quality of the new birth certificate data: validation study in North Carolina. Am J Public Health 1993; 83:1163-5.

20. Reichman NE, Hade EM. Validation of birth certificate data: a study of women in New Jersey's Health Start Program. Ann Epidemiol 2001; 11:186-93.

21. Moraes CL, Reichenheim ME. Validade do exame clínico do recém-nascido para estimação da idade gestacional: uma comparação do escore New Ballard com a data da última menstruação e ultra-sonografia. Cad Saúde Pública 2000; 16:83-94.

22. Piper JM, Mitchel EF, Snowden M, HallC, Taylor P. Validation of 1989 Tennessee birth certificates using maternal and newborn hospital records. Am J Epidemiol 1993; 137:758-68.

23. Costakos DT, Love LA, Kirby RS. The computerized perinatal database: are the data reliable? Am J Perinatol 1998; 15:453-9.

Recebido em 13/Fev/2003

Versão final reapresentado em 24/Set/2003

Aprovado em 14/Jan/2004 